"This accepted author manuscript is copyrighted and published by Elsevier. It is posted here by agreement between Elsevier and MTA. The definitive version of the text was subsequently published in Journal of Molecular Liquids, 205, 9-15, 15 May 2014, doi:10.1016/j.molliq.2014.05.001. Available under license CC-BY-NC-ND."

\title{
Structure of the adsorption layer of various ionic and non- ionic surfactants at the free water surface, as seen from computer simulation and ITIM analysis
}

Nóra Abrankó-Rideg ${ }^{\mathrm{a}}$, George Horvai ${ }^{\mathrm{b}, \mathrm{c}}$, and Pál Jedlovszky ${ }^{\mathrm{a}, \mathrm{b}, \mathrm{d}, *}$

${ }^{a}$ Laboratory of Interfaces and Nanosize Systems, Institute of Chemistry, Eötvös Loránd University, Pázmány P. Stny 1/A, H-1117 Budapest, Hungary

${ }^{\mathrm{b}}$ MTA-BME Research Group of Technical Analytical Chemistry, Szt. Gellért tér 4, H-1111 Budapest, Hungary

${ }^{c}$ Department of Inorganic and Analytical Chemistry, Budapest University of Technology and Economics, Szt. Gellért tér 4, H-1111 Budapest, Hungary

${ }^{\mathrm{d}}$ EKF Department of Chemistry, Leányka u. 6, H-3300 Eger, Hungary

Running title: Structure of surfactants at water

"Electronic mail: pali@chem.elte.hu 


\section{Abstract:}

Molecular dynamics simulations of the adsorption layer of five different surfactant molecules, namely pentanol, octanol, dodecanol, dodecyl trimethyl ammonium chloride, and sodium dodecyl sulphate have been performed at the free surface of water at two different surface densities, namely $1 \mu \mathrm{mol} / \mathrm{m}^{2}$ (corresponding to unsaturated adsorption layer), and $4 \mu \mathrm{mol} / \mathrm{m}^{2}$ (corresponding to saturated adsorption layer), on the canonical ensemble at room temperature. The surfactants have been chosen in such a way that the effect of their headgroup charge as well as alkyl tail length on the properties of the adsorption layer can be separately investigated. The results are analysed in terms of the molecular level structure of the adsorption layer; organisation of the different groups and molecules along the macroscopic surface normal axis as well as conformation and orientation of the apolar tail is investigated in detail. In addition, the roughness of the surface of the aqueous phase is also analysed, using the ITIM method for accurately locating the real, capillary wave corrugated surface of the aqueous phase.

Keywords: Surfactants, liquid/vapor interface, computer simulation, intrinsic surface analysis 


\section{Introduction}

Surfactant molecules of various types are of great importance both in science (e.g., colloid chemistry, surface science) and in industrial applications (e.g., food technology or washing industry). In addition, these kind of molecules, characterised by separate apolar and polar or ionic structural units are of key importance also in living systems, e.g., as the main constituents of the membrane of all eukaryotic cells. Such molecules are extremely surface active; at very low bulk concentration they can already saturate the surface of their aqueous solution. The reason for this behaviour is well understood: these molecules can effectively reduce the surface tension by aligning at the surface in such a way that their polar groups are immersed into the aqueous phase whilst their apolar parts stick out to the vapour phase.

Although the general features of the adsorption layer of surfactants are well known, much less is known about the details of the structure and dynamics of such layers. Thus, questions concerning the time scale of the lateral mobility of the surfactants [1], the immersion depth of their polar part into the aqueous phase [2], the role of the headgroup type (i.e., ionic, non-ionic, zwitterionic, catanionic, etc.) and the number, length, topology and degree of saturation of the apolar tails on the structure and dynamics of the adsorption layer, preferred orientation and conformation of the apolar tails and their dependence on the level of saturation of the adsorption layer have remained unresolved until recently or are still not fully understood.

With the recent development of both various surface sensitive experimental methods and of fast and easily accessible computers, the molecular level properties of surfactant adsorption layers have been studied with increasing intensity in the past two decades both experimentally [3-14] and by computer simulation methods [1,2,15-31]. In fact, computer simulation studies can well complement experimental investigations since, given that a reliable enough model of the real system is used, they can provide a three dimensional insight of atomistic resolution into the structure and dynamics of the system to be studied. Thus, the adsorption layer of various cationic [1,2,18,19,25,30], anionic [1,2,20,21,24,28-31], catanionic [25], zwitterionic [15-17], and non-ionic surfactants [1,2,22-24,26,30] at the surface of their aqueous solutions have been studied several times. However, there is still a need for systematic comparisons of various structural features, e.g., headgroup type or tail length of the surfactants on the properties of their adsorption layers.

In this paper we attempt to partly fill this gap by comparing the (molecular level) structure of the adsorption layer of five different surfactant molecules, namely pentanol (PA), 
octanol (OA), dodecanol (DA), dodecyl trimethyl ammonium chloride (DTAC) and sodium dodecyl sulphate (SDS), at the surface of their aqueous solutions. Three of these surfactants, i.e., PA, OA and DA have the same (alcoholic) headgroup and differ from each other only in their apolar chain lengths. On the other hand, DA, DTAC and SDS all have the same dodecyl tail, but differ in their headgroups, belonging to non-ionic, cationic and anionic surfactants, respectively. The schematic structures of these surfactants are shown in Figure 1. The comparisons are made at two different surface densities, namely at $1 \mu \mathrm{mol} / \mathrm{m}^{2}$ and $4 \mu \mathrm{mol} / \mathrm{m}^{2}$, corresponding to highly unsaturated and saturated adsorption layers, respectively.

In two of our recent studies we investigated two particular features of these systems in detail. Thus, we have shown that the lateral dynamics of these surfactant molecules is very fast; it occurs on a time scale comparable with that of the diffusion of the water molecules [1]. Hence, describing their adsorption isotherm models assuming localized molecules, such as the Langmuir [32-34] or the Frumkin isotherm [34,35] are not applicable. Instead, an adsorption isotherm based on mobile adsorbates, such as the Volmer isotherm [34,36,37], has to be used for such systems [1]. We have also shown that the headgroups of ionic surfactants are immersed into the aqueous phase several molecular layers deep, while those of the alcoholic surfactants stay in the outmost molecular layer of the aqueous phase. [2]

In the present study we focus our attention on the organisation of the various groups along the surface normal axis, orientation and conformation of the alkyl tail, and molecular scale roughness of the surface of these systems. The paper is organised as follows. In section 2 details of the calculations, including both the computer simulations and surface analyses performed are given. The obtained results are presented and discussed in detail in section 3 . Finally, in section 4 the main conclusions of this study are summarised.

\section{Computational details}

The computer simulations on which the present study is based have already been described in detail in our previous publications [1,2], therefore, they are only briefly reminded here. Molecular dynamics simulations of ten different systems, consisting the liquid-vapour interface of water, and one of the five surfactant considered (i.e., PA, OA, DA, DTAC or SDS) have been performed on the canonical $(N, V, T)$ ensemble at $298 \mathrm{~K}$. For each surfactant,

the simulation has been repeated with two surface densities, namely $1 \mu \mathrm{mol} / \mathrm{m}^{2}$ and $4 \mu \mathrm{mol} / \mathrm{m}^{2}$. The temperature of the system has been controlled by the weak coupling 
algorithm of Berendsen et al. [38]. The $X, Y$ and $Z$ edges of the basic simulation box have been $100.0 \AA, 31.41 \AA$ and $31.41 \AA$, respectively, $X$ being the surface normal axis. The basic box has contained 1598 water molecules, 12 or 48 surfactants (in cases of the $1 \mu \mathrm{mol} / \mathrm{m}^{2}$ and $4 \mu \mathrm{mol} / \mathrm{m}^{2}$ systems, respectively), arranged in equal number at the two interfaces in the basic box, and, in the case of DTAC or SDS, the same number of $\mathrm{Cl}^{-}$or $\mathrm{Na}^{+}$counterions, respectively, inside the aqueous phase.

The energy of the system has been calculated as the sum of intra- and intermolecular contributions. The intramolecular energy term included contributions from the bond angle bending and dihedral rotation of the surfactant molecules, described by harmonic and Ryckaert-Bellmans [39] potential functions, respectively. The geometry of the water molecules as well as surfactant bond lengths have been kept unchanged in the simulations by means of the SETTLE [40] and LINCS [41] algorithms, respectively. The intramolecular energy term has been calculated as the sum of the Lennard-Jones and Columbic interaction energies of all atom pairs, except the ones in 1-2 or 1-3 relative positions. The $\mathrm{CH}_{2}$ and $\mathrm{CH}_{3}$ groups have been treated as united atoms. All interactions have been truncated to zero beyond the group-based cut-off distance of $15 \AA$; the long range part of the electrostatic interaction has been accounted for by Ewald summation in its smooth particle mesh (PME) implementation. [42] Surfactants and counterions have been described by the GROMOS force field $[43,44]$, the charge distribution of the headgroup of the DTAC ion has been taken from Schweighofer and Benjamin [45], whereas water molecules have been modelled by the rigid SPC potential [46].

Simulations have been performed by the GROMACS 3.3.2 program package [47]. Equations of motion have been integrated in $1 \mathrm{fs}$ time steps. The systems have been equilibrated for $5 \mathrm{~ns}$. This was followed by the $5 \mathrm{~ns}$ long production stage, during which 1000 equilibrium sample configurations per system, separated by 5 ps long trajectories each, have been dumped for the analyses. Equilibrium snapshots of the surface of all the ten systems are shown in Figure 2.

To determine the molecular scale roughness of the liquid surface one has to properly identify the full set of the surface atoms, i.e., the ones that are right at the boundary of the two phases. For this purpose, we used the Identification of the Truly Interfacial Molecules (ITIM) method [48] with a probe sphere radius of $1.25 \AA$ and a $60 \times 60$ grid of test lines along the interface normal axis $X$. The size of the atoms has been described by their Lennard-Jones distance parameter, $\sigma$. Tail $\mathrm{C}$ atoms have been regarded as part of the aqueous phase only if 
they had a contact water oxygen neighbour, otherwise they were disregarded in the ITIM analysis [2]. For this purpose, tail $\mathrm{C}$ - water $\mathrm{O}$ pairs have been regarded as being in contact with each other if their distance was less than $4.5 \AA$, i.e., the first minimum position of their radial distribution function [2]. The position of the interface along the test lines has been approximated by the position of the probe sphere once it was stopped along this test line by the first atom of the aqueous phase.

\section{Results and discussion}

\subsection{Density profiles}

To analyse the organisation of the molecules along the surface normal axis, $X$, we have calculated the density profile of the water molecules, headgroups, chain terminal $\mathrm{CH}_{3}$ groups and entire alkyl tails of the surfactant molecules, and, in the case of the ionic surfactants, also that of the counterions. The profiles obtained in the $1 \mu \mathrm{mol} / \mathrm{m}^{2}$ and $4 \mu \mathrm{mol} / \mathrm{m}^{2}$ systems are shown in Figures 3 and 4, respectively.

As is seen, the polar headgroups are indeed inward oriented in every case. Further, with the exception of PA, no surfactant molecule entered into the aqueous phase. On the other hand, a detectable but still a very small amount of PA has dissolved in the aqueous phase; the bulk concentration of PA turns out to be about $0.05 \mathrm{M}$ for both surface densities.

It is seen that the distance between the headgroup and tail terminal $\mathrm{CH}_{3}$ density peaks increases with increasing chain length, and this increase becomes larger at higher surface density. Thus, the distance of the position of these two peaks turns out to be $2.7 \AA$ for PA and $4.8 \AA$ for DA at $1 \mu \mathrm{mol} / \mathrm{m}^{2}$, whilst these values are $3.9 \AA$ and $9.8 \AA$, respectively, at $4 \mu \mathrm{mol} / \mathrm{m}^{2}$. Further, this distance is larger for the ionic surfactants, being $7.1-7.3 \AA$ at $1 \mu \mathrm{mol} / \mathrm{m}^{2}$ and $11.3 \AA$ at $4 \mu \mathrm{mol} / \mathrm{m}^{2}$, than for the non-ionic surfactant DA corresponding to the same tail length. These findings suggest that (i) in the case of the saturated adsorption layer the alkyl tails point straighter to the vapour phase than in the unsaturated layers, and (ii) the alkyl tail of the ionic surfactants is either more elongated or points straighter outward than that of the non-ionic ones. These points will be addressed further in the next subsection.

It is also clear that the headgroup of the two ionic surfactants penetrates deeper into the aqueous phase than that of the three alcohols at both surface densities considered. This finding is in a clear accordance with our recent result that ionic headgroups are immersed several molecular layers deep into the aqueous phase, and hence pull several tail $\mathrm{C}$ atoms also 
into this phase, whereas alcoholic $\mathrm{OH}$ groups strongly prefer to stay in the outmost molecular layer of the aqueous phase [2]. At $1 \mu \mathrm{mol} / \mathrm{m}^{2}$ the counterion density peak is located $1.5-2 \AA$ farther from the vapour phase than that of the (charged) headgroups, whereas in the case of the $4 \mu \mathrm{mol} / \mathrm{m}^{2}$ systems the position of the two density peaks coincides, indicating the increasing importance of the energetic over the entropic term of the solvation free energy with increasing surface density.

\subsection{Surface orientation and conformation of the hydrocarbon tails}

To further investigate the structure of the alkyl tails of the surfactants, we have calculated the cosine distribution of the angle $\gamma$, formed by the surface normal vector, $\underline{X}$, pointing to the vapour phase, and the vector $\underline{d}$ pointing along the alkyl tail from the chain terminal $\mathrm{CH}_{3}$ group to the first headgroup atom (i.e., $\mathrm{O}, \mathrm{N}$, and $\mathrm{S}-\mathrm{O}-\mathrm{C}$ bound $\mathrm{O}$ for the alcohols, DTAC, and SDS, respectively), and also the distribution of the alkyl tail length, characterised by the magnitude of the vector $\underline{d}$, in all the ten systems simulated. The resulting distributions are shown in Figures 5 and 6, respectively. The obtained results confirm our conclusions drawn on the basis of the density profiles. Thus, in the case of the unsaturated adsorption layer the alkyl chains prefer tilted orientations relative to the macroscopic plane of the surface. The tilt angle of the preferred alignment (i.e., the one corresponding to the peak position of the $P(\cos \gamma)$ distribution) relative to the surface plane scatters between $25^{\circ}$ and $55^{\circ}$, in the case of surfactants of the same headgroup this angle being larger for longer tails, and for surfactants of the same tail lengths being smaller when the headgroup is charged. In the case of the saturated surface $\left(4 \mu \mathrm{mol} / \mathrm{m}^{2}\right)$ the tail of all surfactants prefer to point straight (i.e., perpendicular to the macroscopic surface plane) away from the aqueous phase; and this preference is noticeably stronger for the ionic than for the non-ionic surfactants.

The $P(d)$ tail length distribution is clearly bimodal for PA and OA at both surface densities considered; a broad peak is followed by a sharp and narrow one at larger distances. This second peak gets smaller with increasing tail length and with decreasing surface density. Thus, for the surfactants having a dodecyl tail only a small remain of it is seen at $4 \mu \mathrm{mol} / \mathrm{m}^{2}$, and it turns into a small shoulder at $1 \mu \mathrm{mol} / \mathrm{m}^{2}$. This large distance peak corresponds to the all-trans, i.e., the longest possible conformation of the tail. Clearly, assuming a given gaucheltrans ratio of all the tail dihedrals, the longer the alkyl chain gets the smaller is the probability that all of its dihedrals are aligned in trans conformation. To confirm this assumption we have calculated the ratio of the occasions when a given dihedral is aligned in 
gauche and trans conformations for all tail dihedrals in all the ten systems simulated. The resulting gaucheltrans ratio, shown in the insets of Fig. 5, turns out to be about 0.5, independent of the type of the surfactant molecule, the position of the dihedral along the chain, and the surface density.

The main, lower distance peak of the $P(d)$ distribution corresponds to various different conformations in which one or more dihedrals of different positions are gauche-aligned. This peak is asymmetric; the peak position is located at larger distances than the mean value, in accordance with the fact that trans alignment is energetically favourable with respect to the gauche one. Also, this peak becomes broader with increasing tail length, reflecting the increasing possibilities of the tail conformation.

A striking feature of the obtained $P(d)$ distributions is that they are almost indistinguishable for surfactants of the same tail length and different headgroups at both surface densities. In other words, the surfactant headgroup type has no influence at all on the tail conformation. This finding also implies that the increased distance of the headgroup and chain terminal $\mathrm{CH}_{3}$ density peaks upon the headgroup gets charged (see Figs. 3 and 4) can exclusively be attributed to the straighter surface orientation of the tails of the ionic than those of the non-ionic surfactants.

Finally, we address here the question whether the orientational and conformational preferences are given by the same molecules, or different sets of molecules contribute to the observed orientational and conformational preferences. For this purpose, we have calculated the bivariate joint distribution of $\cos \gamma$ and $d$ in all the ten systems simulated. The resulting distributions, shown in Figure 7, are always unimodal; the peak shifts to larger $d$ values ("upward") with increasing tail length, to lower $\cos \gamma$ values ("leftward") with increasing surface density, and it is sharper for ionic than for non-ionic surfactants. These findings clearly confirm that the observed orientational and conformational preferences are given by the same set of surfactant molecules in every case.

\subsection{Surface roughness}

The interface of two fluid phases, although smooth and planar on the macroscopic length scale, is always corrugated by capillary waves on the molecular length scale. The characterisation of this roughness is, however, far from being a trivial task. In computer simulations the difficulty stems from the fact that such a characterisation requires the knowledge of the real, capillary wave corrugated intrinsic surface of the phase of interest (or, equivalently, the full list of the atoms that constitute the surface layer of this phase). The 
recent development of various intrinsic surface analysis methods [48-54] opened up the possibility of such investigations in computer simulation studies. However, having the intrinsic surface fully known, the characterisation of its roughness is still not an obvious task. Clearly, such a characterisation requires the use of at least two independent parameters, i.e., a frequency-like and an amplitude-like one. [48] Recently we proposed to fit the mean normal distance of two surface points (i.e., their distance along the macroscopic surface normal axis), $\bar{d}$, as a function of their lateral distance (i.e., distance within the macroscopic plane of the surface), $l$, by the function

$$
\bar{d}=\frac{a \xi l}{a+\xi l}
$$

where $\xi$ and $a$ are the frequency-like and amplitude-like roughness parameters, respectively [55]. More recently we demonstrated that the amplitude parameter defined this way is intimately related to the surface tension [56], confirming also the physical relevance of this description.

To analyse the surface roughness of the ten systems simulated we have determined the $\bar{d}(l)$ roughness curve and the $\xi$ and $a$ roughness parameters in the first three atomic layers of their aqueous phase. The obtained roughness curves are shown in Figure 8, whereas the $\xi$ and $a$ parameters corresponding to the first layer of the ten systems are summarised in Table 1.

As is seen, the roughness curves corresponding to alcoholic surfactants of different chain lengths are very similar to each other, although the increase of the hydrocarbon tail length is accompanied by a slight decrease of the frequency-like and a small increase of the amplitude-like roughness parameter. Also, unlike in the case of homogeneous liquid surfaces [57], no tendentious difference is seen between the roughness curves of the subsequent atomic layers. The most evident difference between the roughness curves obtained in the different systems is clearly that in the case of the saturated surface $\left(4 \mu \mathrm{mol} / \mathrm{m}^{2}\right)$ ionic surfactants lead to a considerable increase of the surface roughness in terms of the amplitude parameter, $a$, with respect to the alcoholic surfactants. This $40-70 \%$ increase of the value of the $a$ parameter is related to the aforementioned immersion of the headgroup of the ionic surfactants into the aqueous phase: surfactant molecules immersed to a different extent into the aqueous phase result in a rougher aqueous surface, and hence in larger amplitude-like parameter values. 


\section{Summary and conclusions}

In this paper we presented a detailed and systematic comparison of the molecular level structure of the adsorption layer of various surfactants at the free water surface, both in the case of the saturated and unsaturated adsorption layer. Thus, both the alkyl tail length and the charge of the headgroup of the surfactants have been varied systematically and independently from each other. The results revealed that all surfactants prefer to point straight away from the aqueous phase by the apolar tail in the case of the saturated adsorption layer, and this preference is stronger if the headgroup of the surfactant bears a net charge. On the other hand, when the adsorption layer is unsaturated, the apolar tails prefer tilted alignment relative to the macroscopic plane of the surface, and this tilt angle is smaller (i.e., the tail gets closer to the surface plane) if the tail is shorter or the headgroup is charged. Apolar tails were found to prefer rather elongated conformations, in which only a few dihedrals are gauche-aligned. The gauche/trans ratio turned out to be about 0.5 , independently from the tail length, headgroup type, and position of the given dihedral along the tail. It was also found that, unlike its orientation, the conformation of the apolar tail is independent from the headgroup type. We found that in the case of the unsaturated layer the counterions prefer to stay a few Angströms farther away from the vapour phase than the charged headgroups, whereas in saturated layers this difference is washed out by the dominance of the energetic over the entropic factor in determining the counterion distribution.

We have also performed, to our knowledge, for the first time, a detailed comparative analysis of the roughness of the surface of the different systems. The most important conclusion drawn in this respect is that in the case of the saturated adsorption layer charged surfactants result in a much rougher aqueous surface (at least, in terms of its amplitude) than non-ionic ones. This finding is in a clear accordance with our former result, namely that the headgroup of the ionic surfactants, unlike that of the alcoholic ones, is immersed several molecular layers deep into the aqueous phase. [2]

Acknowledgements. This project is supported by the Hungarian OTKA Foundation under project No. 104234. P. J. is a Szentágothai János fellow of Hungary, supported by the European Union, co-financed by the European Social Fund in the framework of TÁMOP 4.2.4.A/2-11-1-2012-0001 "National Excellence Program” under grant number A2-SZJÖTOK-13-0030. 


\section{References}

[1] N. A. Rideg, M. Darvas, I. Varga, P. Jedlovszky, Langmuir 28 (2012) 14944

[2] N. Abrankó-Rideg, M. Darvas, G. Horvai, P. Jedlovszky, J. Phys. Chem. B 117 (2013) 8733.

[3] J. R. Lu, E. A. Simister, E. M. Lee, R. K. Thomas, A. R. Rennie, J. Penfold, Langmuir 8 (1992) 1837.

[4] J. C. Conboy, M. C. Messmer, G. L. Richmond, J. Phys. Chem. 100 (1996) 7617.

[5] D. E. Gragson, B. M. McCarty, G. L. Richmond, J. Phys. Chem. 100 (1996) 14272.

[6] C. D. Bain, Curr. Opin. Coll. Interf. Sci. 3 (1998) 287.

[7] Z. X. Li, C. C. Dong, R. K. Thomas, Langmuir 15 (1999) 4392.

[8] C. Stubenrauch, P. A. Albouy, R. von Klitzing, D. Langevin, Langmuir 16 (2000) 3206.

[9] J. R. Lu, R. K. Thomas, J. Penfold, Adv. Coll. Interface Sci. 84 (2000) 143.

[10] A. V. Benderskii, K. B. Eisenthal, J. Phys. Chem. B. 105 (2001) 6698.

[11] T. Gilányi, I. Varga, R. Mészáros, Phys. Chem. Chem. Phys. 6 (2004) 4348.

[12] G. Ma, H. C. Allen, Langmuir 22 (2006) 11267.

[13] Z. Zhang, D. Zheng, Y. Guo, H. Wang, Phys. Chem. Chem. Phys. 11 (2009) 991.

[14] A. Angus-Smyth, R. A. Campbell, C. D. Bain, Langmuir 28 (2012) 12479.

[15] H. Alper, D. Bassolino-Klimas, T. R. Stouch, J. Chem. Phys. 99 (1993) 5547.

[16] S. E. Feller, Y. Zhang, R. W. Pastor, J. Chem. Phys. 103 (1995) 10267.

[17] H. Dominguez, A. M. Smondyrev, M. L. Berkowitz, J. Phys. Chem. B 103 (1999) 9582.

[18] J. Böcker, M. Schlenkirch, P. Bopp, J. Brickmann, J. Phys. Chem. 96 (1992) 9915.

[19] M. Tarek, D. J. Tobias, M. L. Klein, J. Phys. Chem. 99 (1995) 1393.

[20] K. Schweighofer, U. Essman, M. Berkowitz, J. Phys. Chem. B 101 (1997) 3793.

[21] H. Domínguez, M. Berkowitz, J. Phys. Chem. B 104 (2000)5302.

[22] P. Jedlovszky, I. Varga, T. Gilányi, J. Chem. Phys. 120 (2004) 11839.

[23] A. Paszternák, É. Kiss, P. Jedlovszky, J. Chem. Phys. 122 (2005) 124704.

[24] H. Domínguez, M. Rivera, Langmuir 21 (2005) 7257.

[25] J. Rodriguez, E. Clavero, D. Laria, J. Phys. Chem. B 109 (2005) 24427.

[26] J. Chanda, S. Bandyopadhyay, J. Phys. Chem. B 110 (2006) 23482. 
[27] Gy. Hantal, L. B. Pártay, I. Varga, P. Jedlovszky, T. Gilányi, J. Phys. Chem. B 111 (2007) 1769.

[28] H. Martínez, E. Chacón, P. Tarazona, F. Bresme, Proc. R. Soc. A 467 (2011) 1939.

[29] Darvas, M.; Gilányi, T.; Jedlovszky, J. Phys. Chem. B 115 (2011) 933.

[30] J. Pang, Y. Wang, G. Xu, G.; T. Han, J. Phys. Chem. B 115 (2011) 2518.

[31] R. Vacha, S. Roke, J. Phys. Chem. B 116 (2012) 11936.

[32] J. Langmuir, J. Am. Chem. Soc. 38 (1916) 2221.

[33] D. J. Shaw, Introduction to Colloid and Surface Chemistry, Butterworths, London, 1980.

[34] Lyklema, J, Fundamentals of Interface and Colloid Science, Academic Press, London, 2000 .

[35] A. Frumkin, Z. Phys. Chem. 116 (1925) 466.

[36] M. Volmer, Trans. Faraday Soc.28 (1932) 359.

[37] M. Volmer, P. Mahnert, Z. Phys. Chem. 115 (1925) 239.

[38] H. J. C. Berendsen, J. P. M. Postma, A. DiNola, J. R. Haak, J. Chem. Phys.81 (1984) 3684.

[39] J. P. Ryckaert, A. Bellemans, Faraday Discuss. Chem. Soc. 66 (1978) 95.

[40] S. Miyamoto, P. A. Kollman, J. Comp. Chem. 13 (1992) 952.

[41] B. Hess, H. Bekker, H. J. C. Berendsen, J. G. E. M. Fraaije, J. Comp. Chem. 18 (1997)

[42] U. Essman, L. Perera, M. L. Berkowitz, T. Darden, H. Lee, L. G. Pedersen, J. Chem. Phys. 103 (1995) 8577.

[43] J. Hermans, H. J. C. Berendsen, W. F van Guntseren, J. P. M. Postma, Biopolymers 23 (1984) 1513.

[44] W. F van Guntseren, H. J. C. Berendsen, Groningen Molecular Simulation (GROMOS) Library Manual, Biomos, Groningen, 1987.

[45] K. Schweighofer, I. Benjamin, J. Phys. Chem. A 103 (1999) 10274.

[46] H. J. C. Berendsen, J. P. M. Postma, W. F. van Gunsteren, J. Hermans, in: B. Pullman (Ed.), Intermolecular Forces, Reidel, Dordrecht, 1981, p. 331.

[47] E. Lindahl, B. Hess, D. van der Spoel, J. Mol. Mod. 7 (2001) 306.

[48] L. B. Pártay, G. Hantal, P. Jedlovszky, Á. Vincze, G. Horvai, J. Comp. Chem. 29 (2008) 945.

[49] E. Chacón, P. Tarazona, Phys. Rev. Lett. 91 (2003) 166103.

[50] J. Chowdhary, B. M. Ladanyi, J. Phys. Chem. B 110 (2006) 15442.

[51] M. Jorge, M. N. D. S. Cordeiro, J. Phys. Chem. C 111 (2007) 17612. 
[52] A. P. Wilard, D. Chandler, J. Phys. Chem. B 114 (2010) 1954.

[53] M. Jorge, P. Jedlovszky, M. N. D. S. Cordeiro, J. Phys. Chem. C 114 (2010) 11169.

[54] M. Sega, S. S. Kantorovich, P. Jedlovszky M. Jorge, J. Chem. Phys., 138 (2013) 044110 .

[55] M. Darvas, L. B. Pártay, P. Jedlovszky, G. Horvai, J. Mol. Liq. 153 (2010) 88.

[56] P. Jedlovszky, M. Darvas, G. Horvai, Z. Naturforsch. 68a (2013) 123.

[57] G. Hantal, M. Darvas, L. B. Pártay, G. Horvai, P. Jedlovszky, J. Phys.: Condens. Matter 22 (2010) 284112. 


\section{Tables}

Table 1. Roughness parameters of the first atomic layer of the aqueous phase in the ten systems simulated.

\begin{tabular}{cccccc}
\hline \hline \multirow{2}{*}{ surfactant } & \multicolumn{3}{c}{$1 \mu \mathrm{mol} / \mathrm{m}^{2}$} & & \multicolumn{2}{c}{$4 \mu \mathrm{mol} / \mathrm{m}^{2}$} \\
\cline { 2 - 3 } \cline { 5 - 6 } & $\xi$ & $a / \AA$ & & $\xi$ & $a / \AA$ \\
\hline PA & 1.09 & 2.70 & & 1.12 & 2.52 \\
OA & 1.06 & 2.82 & & 1.09 & 2.50 \\
DA & 1.03 & 2.99 & & 1.01 & 2.73 \\
SDS & 1.02 & 2.60 & & 0.94 & 3.89 \\
\hline \hline
\end{tabular}




\section{Figure legends}

Fig. 1. Schematic structure of the five surfactant molecules considered. The $\mathrm{C}, \mathrm{O}, \mathrm{H}, \mathrm{S}$ and $\mathrm{N}$ atoms as well as the $\mathrm{Na}^{+}$and $\mathrm{Cl}^{-}$counterions are shown by grey, red, white, yellow, light blue, dark blue and green colours, respectively. $\mathrm{H}$ atoms linked to $\mathrm{C}$ atoms are omitted for clarity.

Fig. 2. Equilibrium snapshot (in top view) of the ten systems, as taken out from the simulations. Top row: systems of $1 \mu \mathrm{mol} / \mathrm{m}^{2}$ surface density, bottom row: systems of $4 \mu \mathrm{mol} / \mathrm{m}^{2}$ surface density. Water $\mathrm{O}, \mathrm{C}$, surfactant $\mathrm{O}$, surfactant headgroup $\mathrm{H}, \mathrm{S}$ and $\mathrm{N}$ atoms as well as the $\mathrm{Na}^{+}$and $\mathrm{Cl}^{-}$ counterions are shown by green, grey, red, white, yellow, blue, magenta and brown colours, respectively. For clarity, water hydrogens as well as surfactant hydrogens chemically linked to a C atom are omitted, and surfactant atoms and counterions are shown by space filling representation.

Fig. 3. Density profile of the water molecules (black squares), all the $\mathrm{C}$ atoms of the hydrocarbon chains (red circles), chain terminal methyl groups (green up triangles), headgroup central atoms (i.e., $\mathrm{O}, \mathrm{N}$ and S for alcohols, DTAC and SDS, respectively; blue down triangles) and counterions (orange lines) as calculated in the systems containing PA (top panel), OA (second panel), DA (third panel), DTAC (fourth panel) and SDS (bottom panel) at the surface density of $1 \mu \mathrm{mol} / \mathrm{m}^{2}$. The scales on the left refer to the water, whilst those on the right to all the other profiles. All the profiles shown are symmetrised over the two liquid surfaces present in the basic simulation box.

Fig. 4. Density profile of the water molecules (black squares), all the $\mathrm{C}$ atoms of the hydrocarbon chains (red circles), chain terminal methyl groups (green up triangles), headgroup central atoms (i.e., $\mathrm{O}, \mathrm{N}$ and S for alcohols, DTAC and SDS, respectively; blue down triangles) and counterions (orange lines) as calculated in the systems containing PA (top panel), OA (second panel), DA (third panel), DTAC (fourth panel) and SDS (bottom panel) at the surface density of $4 \mu \mathrm{mol} / \mathrm{m}^{2}$. The scales on the left refer to the water and hydrocarbon chain, whilst those on the right to all the other profiles. All the profiles shown are symmetrised over the two liquid surfaces present in the basic simulation box. 
Fig. 5. Cosine distribution of the angle $\gamma$, formed by the vector pointing from the tail terminal $\mathrm{CH}_{3}$ group to the first headgroup atom, and the surface normal axis pointing away from the aqueous phase, as obtained in the systems containing PA (solid black lines), OA (dashed red lines), DA (dotted blue lines), DTAC (dash-dotted magenta lines), and SDS (dash-dot-dotted green lines) at the surface densities of $1 \mu \mathrm{mol} / \mathrm{m}^{2}$ (bottom panel) and $4 \mu \mathrm{mol} / \mathrm{m}^{2}$ (top panel). The insets show the gaucheltrans ratio of the different dihedrals in the different systems.

Fig. 6. Distribution of the alkyl tail length, characterised by the distance $d$ of the tail terminal $\mathrm{CH}_{3}$ group from the first headgroup atom, as obtained in the systems containing PA (solid black lines), OA (dashed red lines), DA (dotted blue lines), DTAC (dash-dotted magenta lines), and SDS (dashdot-dotted green lines) at the surface densities of $1 \mu \mathrm{mol} / \mathrm{m}^{2}$ (bottom panel) and $4 \mu \mathrm{mol} / \mathrm{m}^{2}$ (top panel).

Fig. 7. Bivariate distribution of the orientation and length of the alkyl tail, characterised by the cosine of the angle $\gamma$ (formed by the vector pointing from the tail terminal $\mathrm{CH}_{3}$ group to the first headgroup atom, and the surface normal axis pointing away from the aqueous phase), and the distance $d$ of tail terminal $\mathrm{CH}_{3}$ group and first headgroup atom, as obtained in the systems containing PA (first column), OA (second column), DA (third column), DTAC (fourth column), and SDS (fifth column) at the surface densities of $1 \mu \mathrm{mol} / \mathrm{m}^{2}$ (top row) and $4 \mu \mathrm{mol} / \mathrm{m}^{2}$ (bottom row). Lighter shades of grey indicate higher probabilities.

Fig. 8. Average lateral distance of two surface points as a function of their lateral distance in the $1 \mu \mathrm{mol} / \mathrm{m}^{2}$ (left) and $4 \mu \mathrm{mol} / \mathrm{m}^{2}$ (right) systems containing DA (top), DTAC (middle) and SDS (bottom). Roughness curves corresponding to the first, second and third layers are marked by black, red and blue colours, respectively. The insets compare the roughness curves of the first layer of systems containing PA (magenta triangles), OA (green squares) and DA (black circles) in the surface densities of $1 \mu \mathrm{mol} / \mathrm{m}^{2}$ (left inset) and $4 \mu \mathrm{mol} / \mathrm{m}^{2}$ (right inset). 
Figure 1.

Abrankó-Rideg et al.
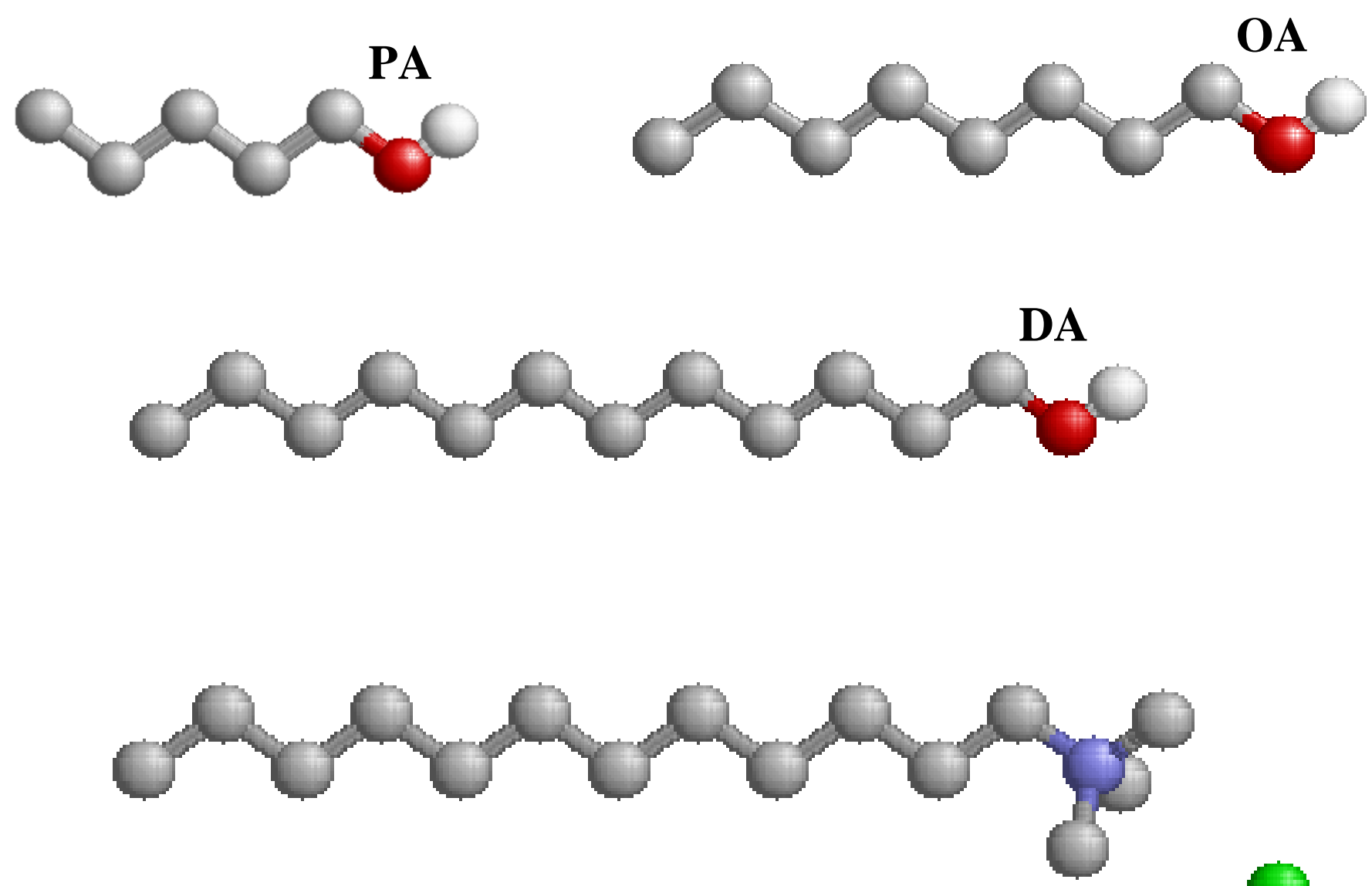

DTAC

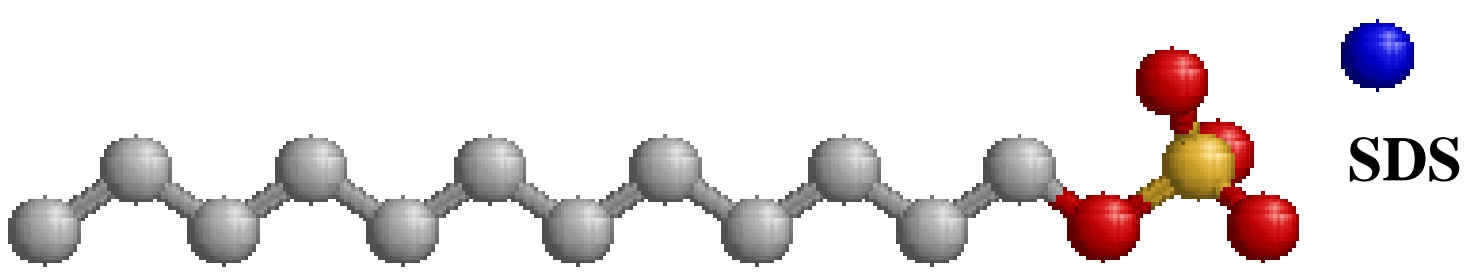


Figure 2.

Abrankó-Rideg et al.

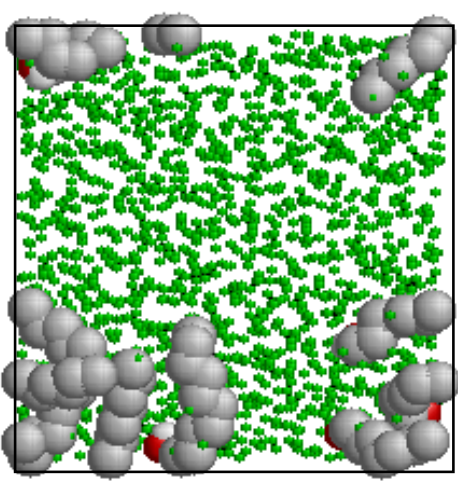

DA

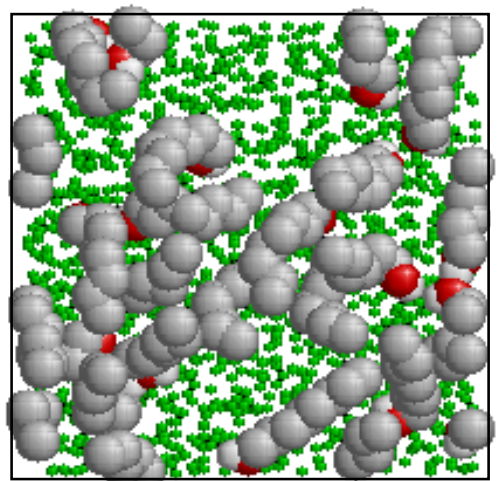

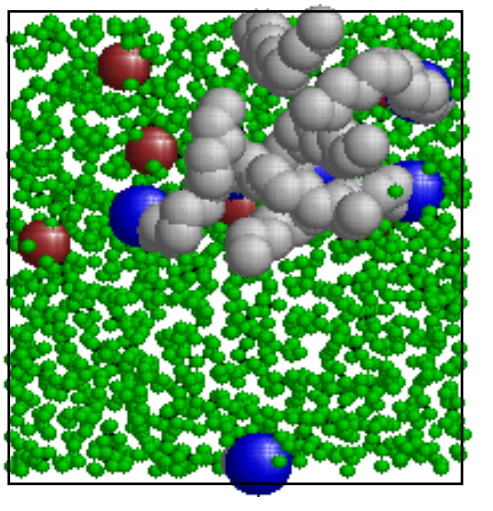

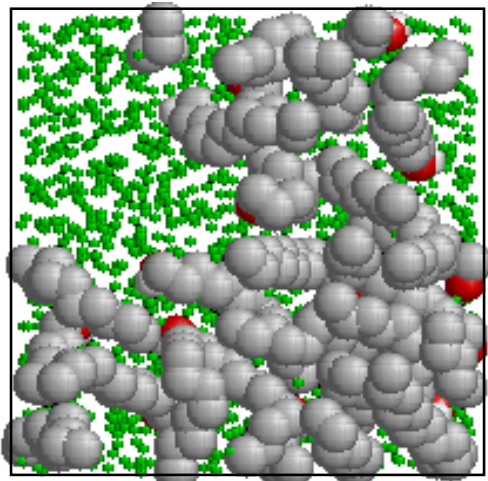

DTAC

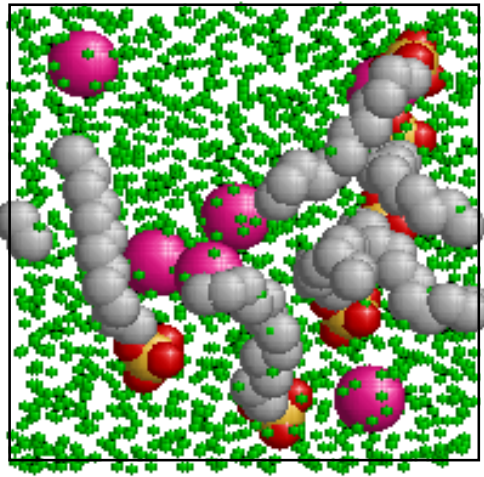

SDS
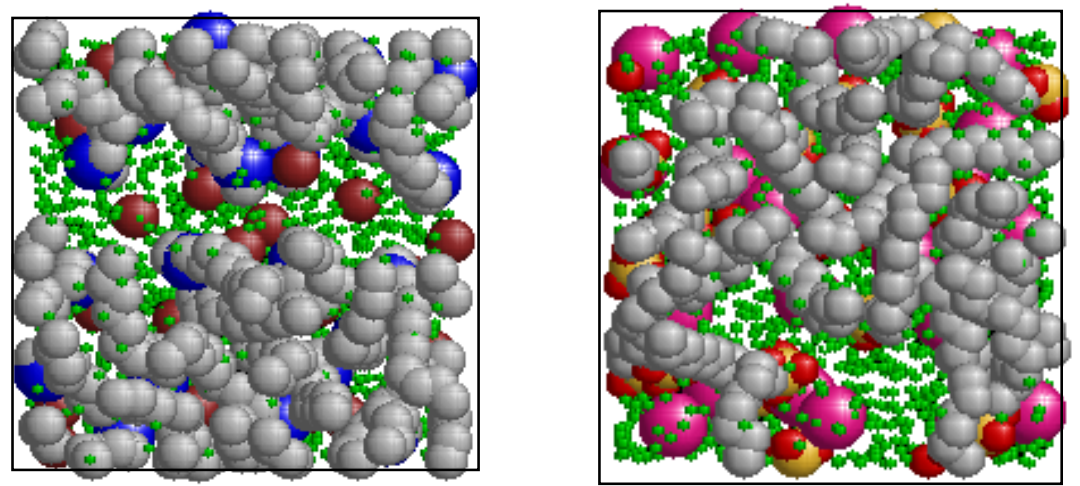
Figure 3.

Abrankó-Rideg et al.

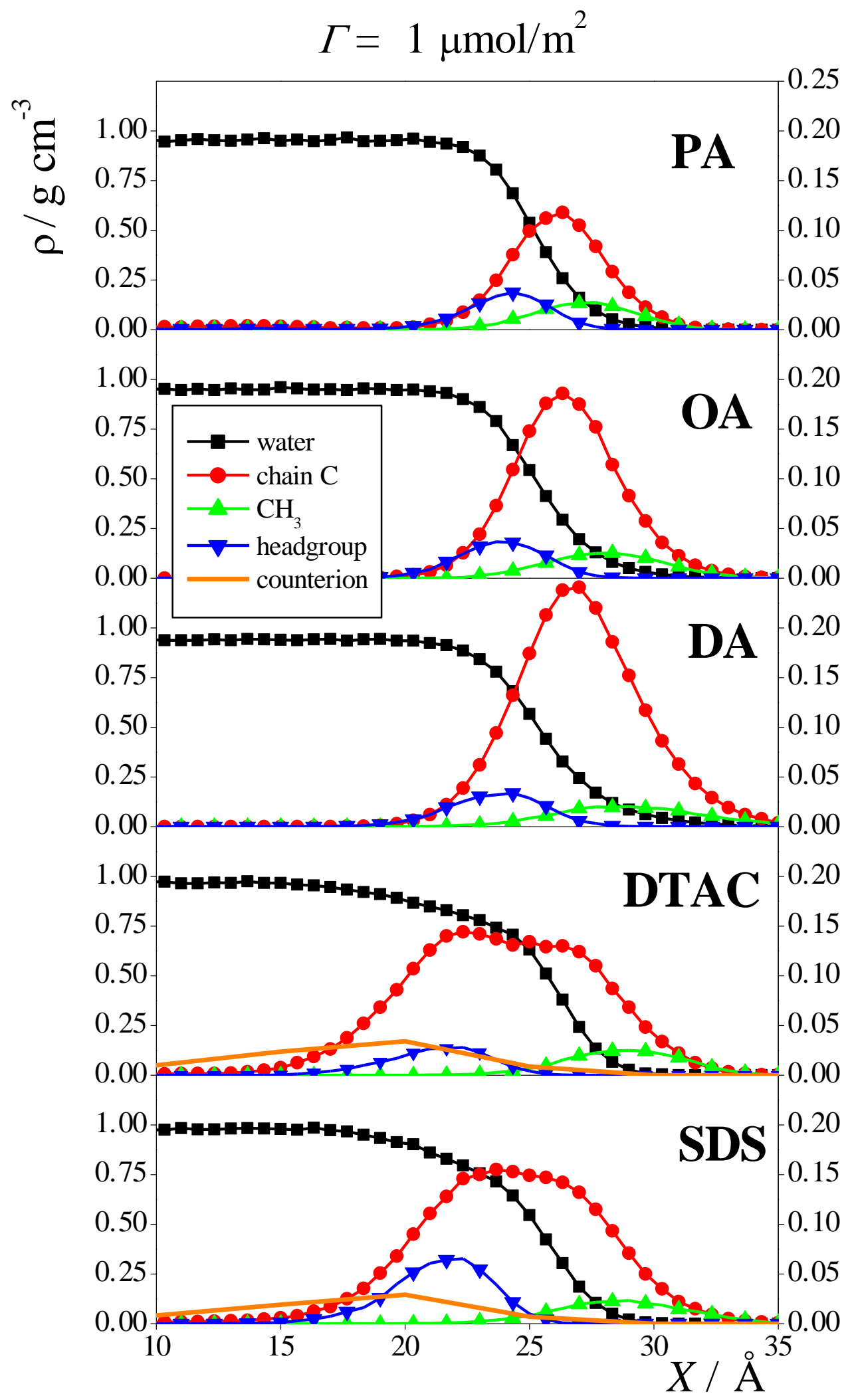


Figure 4.

Abrankó-Rideg et al.

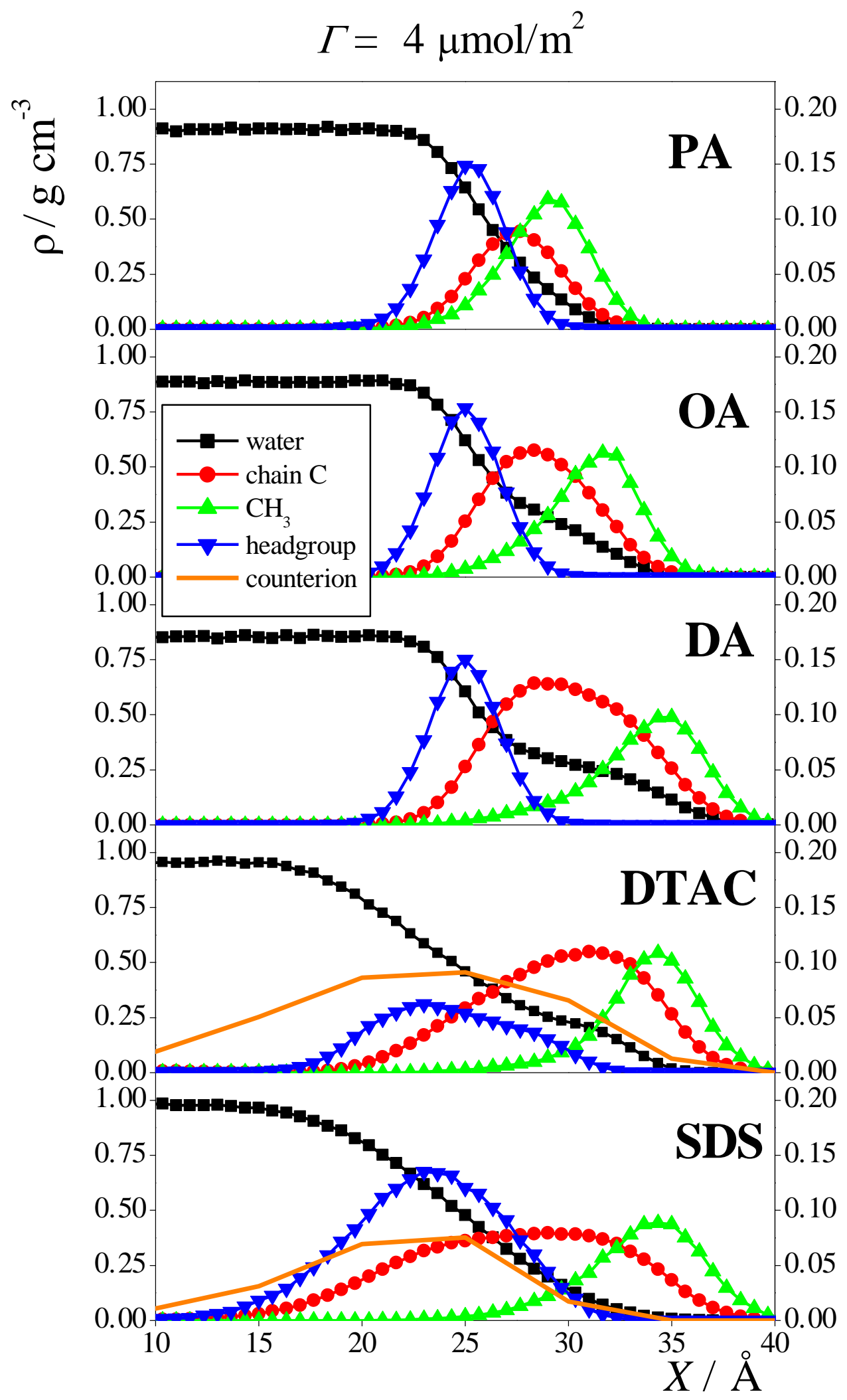


Figure 5.

Abrankó-Rideg et al.

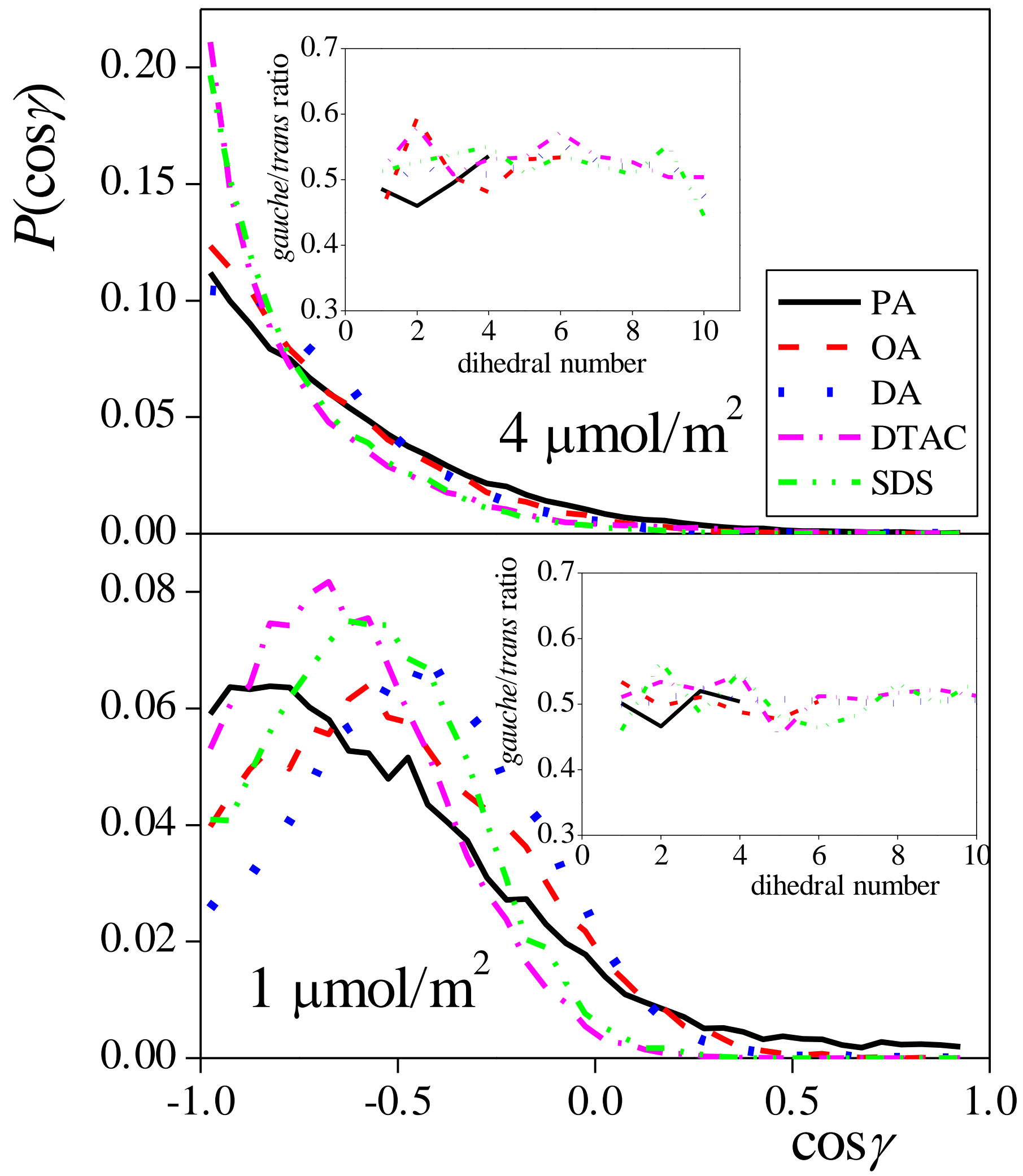


Figure 6.

Abrankó-Rideg et al.

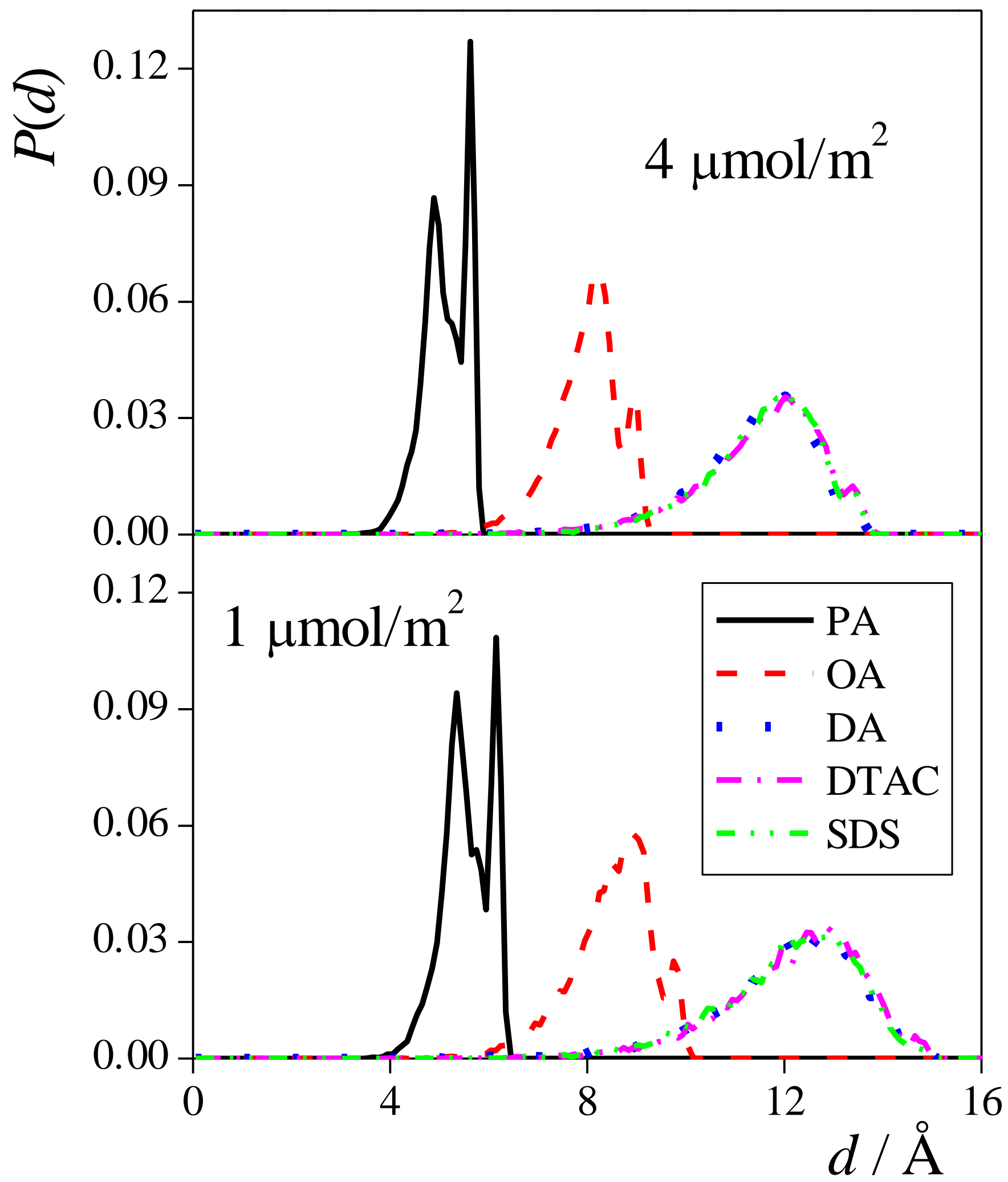


Figure 7.

Abrankó-Rideg et al.

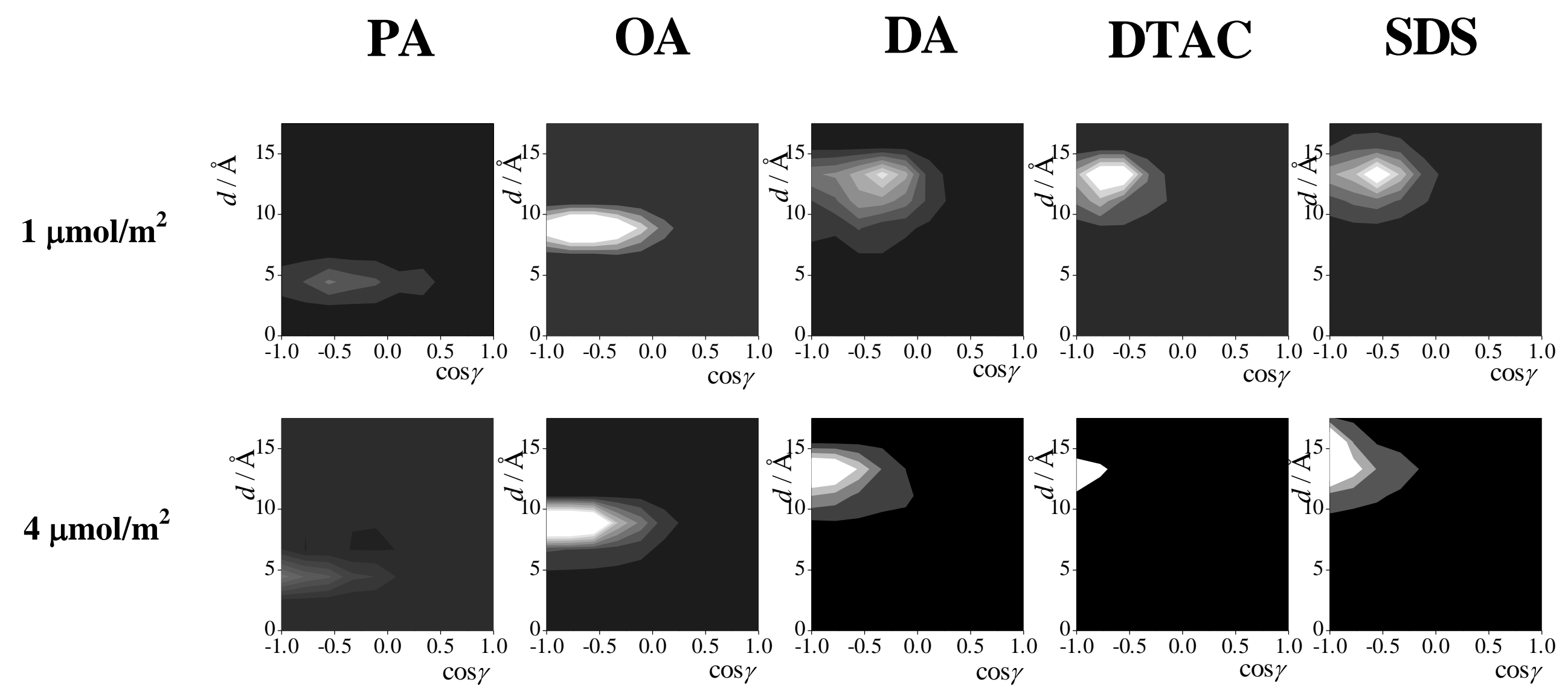


Figure 8.

Abrankó-Rideg et al.

\section{$1 \mu \mathrm{mol} / \mathrm{m}^{2} \quad 4 \mu \mathrm{mol} / \mathrm{m}^{2}$}

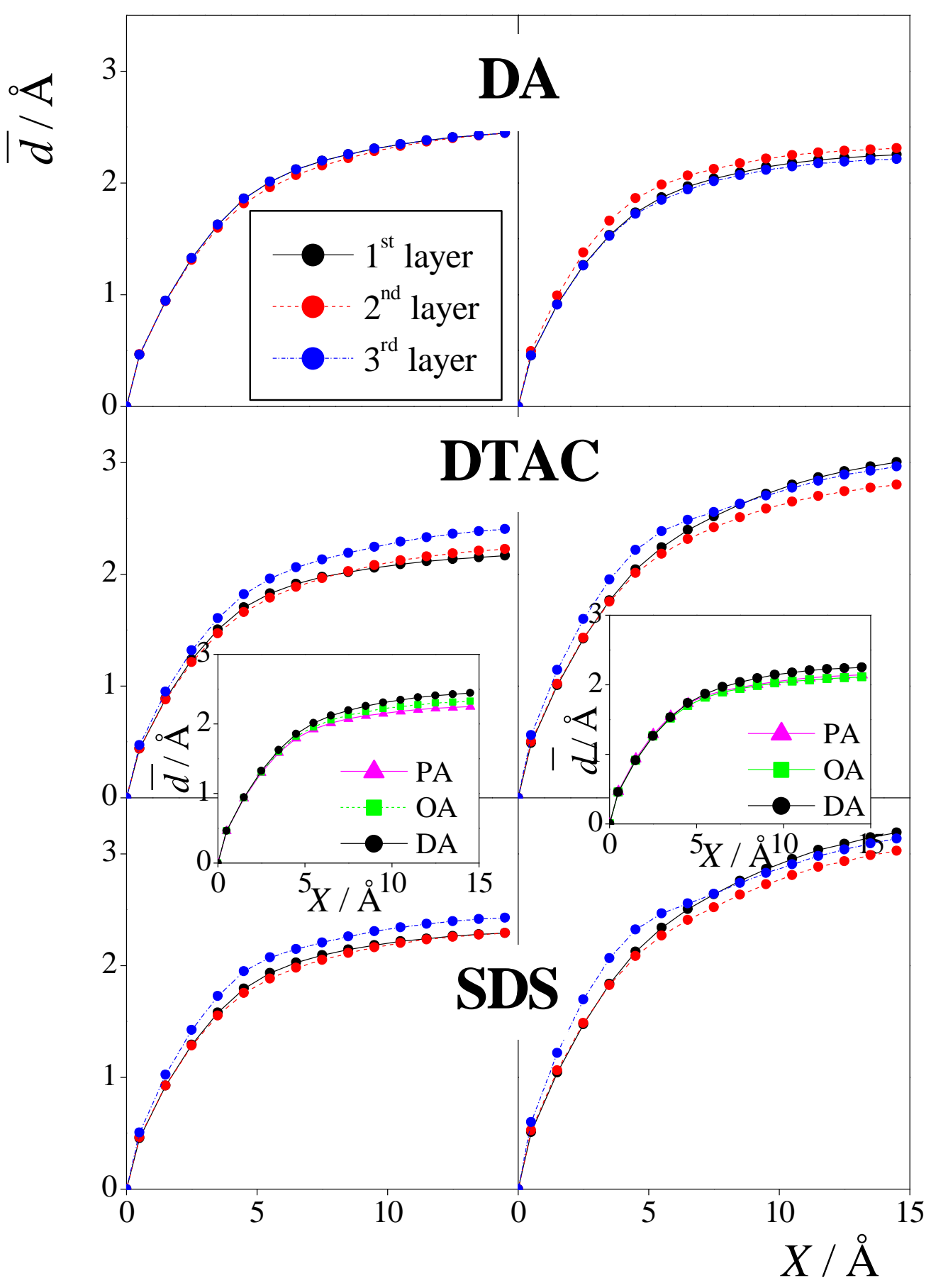

